manuscrlpta math. $77,169-189$ (1992)

\title{
ISOPERIMETRIC INEQUALITIES ON MINIMAL SUBMANIFOLDS OF SPACE FORMS
}

\section{Jaigyoung Choe* and Robert Gulliver}

\begin{abstract}
For a domain $U$ on a certain $k$-dimensional minimal submanifold of $S^{n}$ or $H^{n}$, we introduce a "modified volume" $M(U)$ of $U$ and obtain an optimal isoperimetric inequality for $U k^{k} \omega_{k} M(D)^{k-1} \leq \operatorname{Vol}(\partial D)^{k}$, where $\omega_{k}$ is the volume of the unit ball of $\mathbf{R}^{k}$. Also, we prove that if $D$ is any domain on a minimal surface in $S_{+}^{n}$ (or $H^{n}$ respectively), then $D$ satisfies an

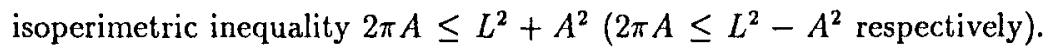
Moreover, we show that if $U$ is a $k$-dimensional minimal submanifold of $H^{n}$, then $(k-1) \operatorname{Vol}(U) \leq \operatorname{Vol}(\partial U)$.
\end{abstract}

Let $C$ be a simple closed curve in the plane, bounding the domain $D$. Let the length of $C$ be $L$ and the area of $D$ be $A$. Then the classical isoperimetric inequality states that

$$
4 \pi A \leq L^{2}
$$

with equality if and only if $C$ is a circle. If $D$ is a domain on the sphere, the sharp isoperimetric inequality for $D$ was given by F. Bernstein [2]:

$$
4 \pi A \leq L^{2}+K L^{2}
$$

where $K$ is the Gauss curvature of the sphere. In fact, in this form it is valid both for the sphere and for the plane, where $K \equiv 0$. One might guess that it would hold equally for the hyperbolic plane $H^{2}$, where $K \equiv-1$. Schmidt [9] showed that this turns out to be the case.

\footnotetext{
'Supported in part by KME and GARC
} 


\section{Choe - Gulliver}

Considering that minimal surfaces are viewed as generalized planes, it is tempting to conjecture that these sharp isoperimetric inequalities should still hold for domains on any immersed minimal surfaces in space forms, $\mathbf{R}^{n}, S^{n}, H^{n}$. The truth of this conjecture is not yet known, except in special cases. For example, it was shown for minimal surfaces in $\mathbf{R}^{n}$ with radially connected boundary in [4] and later for analogous minimal surfaces in $H^{n}$ in our companion paper [5].

In this paper we introduce a modified volume $M(D)$ of a domain $D$ and obtain an isoperimetric inequality without the additional term that Bernstein and Schmidt had:

$$
k^{k} \omega_{k} M(D)^{k-1} \leq \operatorname{Volume}(\partial D)^{k},
$$

where $\omega_{k}$ is the volume of the unit ball of $\mathbf{R}^{k}$, and $D$ is a domain on a certain $k$-dimensional minimal submanifold of a space form. This modified isoperimetric inequality is proved under any of the following conditions: (Theorem 4) $\partial D$ lies on a geodesic sphere of the space form; (Theorem 2) $k=2, D \subset S_{+}^{n}$ (the hemisphere) and $\partial D$ is weakly connected; (Theorem 1) $k=2, D \subset S_{+}^{n}$ and $\partial D$ is radially connected about a point of $D$. Observe that $D$ is not assumed to have minimum volume. The inequality is sharp for a geodesic ball in a totally geodesic submanifold $\Sigma$, and appears to be a plausible conjecture for any minimal submanifold of $S_{+}^{n}$ or $\mathbf{R}^{n}$.

While we need an assumption of radial connectivity for a partial solution of the above conjectures, L. Simon (see [3, p.318], [8, Theorem 5.3]) made no connectedness assumption and obtained a weaker isoperimetric inequality

$$
2 \pi A \leq L^{2}
$$

for any minimal surface in $\mathbf{R}^{n}$. In the second part of this paper we extend his method and derive (Theorem 5)

$$
2 \pi A \leq L^{2}+A^{2}
$$

for a minimal surface in $S^{n}$, and

$$
2 \pi A \leq L^{2}-A^{2}
$$

for a minimal surface in $H^{n}$. We further extend this method and show (Theorem 6) that if $\Sigma$ is a $k$-dimensional minimal submanifold of $H^{n}$, then

$$
(k-1) \text { Volume }(\Sigma) \leq \text { Volume }(\partial \Sigma)
$$


This is an exact extension of Yau's result for a domain in $H^{k}$ [10]. More isoperimetric inequalities of this kind are proved. Finally, to each isoperimetric inequality of these types, we obtain corresponding Sobolev-type inequalities for smooth nonnegative functions with compact support on minimal surfaces (see Corollaries 1,2, and $3)$.

We would like to thank Walter Wei for bringing Simon's work to our attention.

\section{Modified volume}

We assume throughout this paper that $D$ and $\Sigma$ are differentiable up to its boundary, and that their closures are compact.

Definition 1 Let $p$ be a point in the $n$-dimensional sphere $S^{n}$ and let $r(x)$ be the distance from $p$ to $x$ in $S^{n}$. Given a $k$-dimensional submanifold $\Sigma$ in $S^{n}$, the modified volume of $\Sigma$ with center at $p$ is defined to be

$$
M_{p}(\Sigma)=\int_{\Sigma} \cos r
$$

Similarly for $\Sigma$ in the $n$-dimensional hyperbolic space $H^{n}$, we define the modified volume of $\Sigma$ by

$$
M_{p}(\Sigma)=\int_{\Sigma} \cosh r
$$

Obviously we have in $S^{n}$

$$
M_{p}(\Sigma) \leq \operatorname{Volume}(\Sigma)
$$

and in $H^{n}$

$$
M_{p}(\Sigma) \geq \operatorname{Volume}(\Sigma)
$$

Lemma 1 (a) Suppose that $S^{\mathbf{n}}$ is embedded in $\mathrm{R}^{n+1}$ with $p$ the north pole $(0, \ldots, 0,1)$. Then the modified volume of a domain $U \subset S^{n}$ is the Euclidean volume of the orthogonal projection of $U$ into the horizontal hyperplane $x_{n+1}=0$, counting orientation.

(b) In case $U$ is a domain in $H^{n}$, embed $H^{n}$ isometrically onto the hypersurface $S_{1} x_{1}^{2}+\cdots+x_{n}^{2}-x_{n+1}^{2}=-1, x_{n+1}>0$, of $\mathbf{R}^{n+1}$ with the Minkowski metric $d s^{2}=d x_{1}^{2}+\cdots+d x_{n}^{2}-d x_{n+1}^{2}$ such that $p$ becomes the point $(0, \ldots, 0,1) \in S$. Then $M_{p}(U)$ equals the Euclidean volume of the projection of $U$ onto the hyperplane $x_{n+1}=0$. 


\section{Choe - Gulliver}

Proof. Part (a) follows from the fact that $\cos r$ is the Jacobian of the projection of $S^{n}$ into $x_{n+1}=0$. For part $(b)$, since the metric on $H^{n}$ and the distance function $r$ are rotationally symmetric about $p$, it suffices to consider only the case where $U \subset H^{1}$. Let $x=\left(x_{1}, x_{2}\right) \in H^{1}, x_{1}^{2}-x_{2}^{2}=-1$. Then $x_{1} d x_{1}=x_{2} d x_{2}$ and hence

$$
\begin{aligned}
r(x) & =\int_{p}^{x} d s=\int_{0}^{x_{1}} \sqrt{d x_{1}^{2}-d x_{2}^{2}}=\int_{0}^{x_{1}} \sqrt{1-\left(x_{1} / x_{2}\right)^{2}} d x_{1} \\
& =\int_{0}^{x_{1}} \frac{1}{\sqrt{1+x_{1}^{2}}} d x_{1}=\log \left(x_{1}+\sqrt{1+x_{1}^{2}}\right) .
\end{aligned}
$$

Hence $x_{1}=\sinh r$. Therefore

$$
d x_{1}=\cosh r d r
$$

which gives the desired result.

In the following lemma, as usual, the notation $V f$, a vector (field) $V$ followed by a function $f$, implies the directional derivative of $f$ in the direction $V$.

Lemma 2 Let $f$ be a smooth function on an $n$-dimensional Riemannian manifold $M$ and $\Sigma$ a k-dimensional submanifold of $M$. Let $\bar{\nabla}$ and $\bar{\Delta}$ be the connection and Laplacian on $M$ respectively, and $\Delta$ the Laplacian on $\Sigma$. If $H$ is the mean curvature vector of $\Sigma$ in $M$, then

$$
\Delta(f \mid \Sigma)=(\bar{\Delta} f) \mid \Sigma+H f-\sum_{\alpha=k+1}^{n} \bar{\nabla}^{2} f\left(\bar{e}_{\alpha}, \bar{e}_{\alpha}\right)
$$

where $\bar{e}_{k+1}, \ldots, \bar{e}_{n}$ are orthonormal vectors which are perpendicular to $\Sigma$.

Proof. Let $e_{1}, \ldots, e_{k}$ be orthonormal vector fields on a neighborhood of a point of $\Sigma$. These vector fields can be smoothly extended to orthonormal vector fields $\bar{e}_{1}, \ldots, \bar{e}_{n}$ on $M$ such that $\bar{e}_{i}=e_{i}, 1 \leq i \leq k$, on $\Sigma$. Then $\sum_{i=1}^{k} \bar{\nabla}_{\bar{e}_{1}} \bar{e}_{i}=H+\sum_{i=1}^{k} \nabla_{e_{i}} e_{i}$, and hence we have

$$
\begin{aligned}
\Delta f & =\sum_{i=1}^{k}\left(e_{i} e_{i} f-\nabla_{e_{i}} e_{i} f\right) \\
& =\sum_{i=1}^{n} \bar{e}_{i} \bar{e}_{i} f-\sum_{\alpha=k+1}^{n} \bar{e}_{\alpha} \bar{e}_{\alpha} f-\sum_{i=1}^{n} \bar{\nabla}_{\bar{e}_{i}} \bar{e}_{i} f+H f+\sum_{\alpha=k+1}^{n} \bar{\nabla}_{\bar{e}_{\alpha}} \bar{e}_{\alpha} f \\
& =\bar{\Delta} f+H f-\sum_{\alpha=k+1}^{n} \bar{\nabla}^{2} f\left(\bar{e}_{\alpha}, \bar{e}_{\alpha}\right)
\end{aligned}
$$




\section{Choe - Gulliver}

Lemma 3 Let $\bar{\nabla}$ and $g$ be the connection and metric tensor respectively of $S^{n}$ or $H^{n}$. On $S^{n}, \bar{\nabla}^{2} \cos r=-(\cos r) g$. On $I^{n}, \bar{\nabla}^{2} \cosh r=(\cosh r) g$.

Proof. Let $p$ be the point from which the distance $r$ is measured. Let $\frac{\partial}{\partial r}, V_{1}, \ldots, V_{n-1}$ be perpendicular vector fields on a neighborhood of $q \in S^{n}$ such that $\left|V_{i}\right|=\sin r$, that $\bar{\nabla}_{V_{i}} V_{j}(q)$ is parallel to $\frac{\partial}{\partial r}$, and that $V_{i}$ are Jacobi fields along any geodesic through $p$. Clearly $\bar{\nabla}_{V_{i}} \frac{\partial}{\partial r}$ is parallel to $V_{i}$ and $\left[V_{i}, \frac{\partial}{\partial r}\right]=0$. Hence

$$
\left\langle\bar{\nabla} V_{i} \frac{\partial}{\partial r}, V_{i}\right\rangle=\left\langle\bar{\nabla}_{\partial / \partial r} V_{i}, V_{i}\right\rangle=\frac{1}{2} \frac{\partial}{\partial r}\left\langle V_{i}, V_{i}\right\rangle=\sin r \cos r
$$

and $\bar{\nabla}_{V_{i}} \frac{\partial}{\partial r}=\cot r V_{i}$. So

$$
\left\langle\bar{\nabla}_{V_{i}} V_{j}, \frac{\partial}{\partial r}\right\rangle=-\left\langle V_{j}, \bar{\nabla}_{V_{i}} \frac{\partial}{\partial r}\right\rangle=-\delta_{i j} \sin r \cos r,
$$

where $\delta_{i j}$ is the Kronecker delta. Hence $\bar{\nabla}_{V_{i}} V_{j}(q)=-\delta_{i j} \sin r \cos r \frac{\partial}{\partial r}$. Therefore

$$
\begin{aligned}
\bar{\nabla}^{2} \cos r\left(V_{i}, V_{j}\right)(q) & =V_{i} V_{j} \cos r-\bar{\nabla}_{V_{i}} V_{j} \cos r \\
& =-\delta_{i j} \sin ^{2} r \cos r=-\cos r g\left(V_{i}, V_{j}\right) .
\end{aligned}
$$

Moreover,

$$
\begin{aligned}
\bar{\nabla}^{2} \cos r\left(\frac{\partial}{\partial r}, V_{j}\right)(q) & =\frac{\partial}{\partial r} V_{j} \cos r-\bar{\nabla}_{\frac{\partial}{\partial r}} V_{j} \cos r \bar{\nabla}_{V} \frac{\partial}{\partial r} \cos r \\
& =\sin r \cos r V_{j}(\cos r)=0=-\cos r g\left(\frac{\partial}{\partial r}, V_{j}\right) .
\end{aligned}
$$

Also,

$$
\begin{aligned}
\bar{\nabla}^{2} \cos r\left(\frac{\partial}{\partial r}, \frac{\partial}{\partial r}\right) & =\frac{d^{2}}{d r^{2}} \cos r-\bar{\nabla}_{\partial / \partial r} \frac{\partial}{\partial r} \cos r \\
& =-\cos r=-\cos r g\left(\frac{\partial}{\partial r}, \frac{\partial}{\partial r}\right) .
\end{aligned}
$$

Thus we have the proof for $S^{n}$. A similar proof holds for $H^{n}$.

Lemma 4 (a) If $\Sigma \subset S^{n}$ is a $k$-dimensional minimal submanifold or a cone, and $r$ is the distance in $S^{n}$ from a fixed point, then

$$
\Delta \cos r=-k \cos r
$$

where $\Delta$ is the Laplacian on the submanifold $\Sigma$ and, in case $\Sigma$ is a cone, $r$ is the distance from the center of $\Sigma$.

(b) Suppose $\Sigma$ is a $k$-dimensional minimal submanifold or a cone in $H^{n}$. Then

$$
\Delta \cosh r=k \cosh r
$$

Ilere again, in case of a cone $\Sigma, r$ is the distance from the center of $\Sigma$. 


\section{Choe - Gulliver}

Proof. The proof for a minimal submanifold follows from Lemma 2 and Lemma 3. On a cone $\Sigma \subset H^{n}$, we see from Lemma 2 that

$$
\begin{aligned}
\Delta \cosh r & =\bar{\Delta} \cosh r+H \cosh r-\sum_{\alpha=k+1}^{n} \bar{\nabla}^{2} \cosh r\left(\bar{e}_{\alpha}, \bar{e}_{\alpha}\right) \\
& =n \cosh r+\sinh r H r-(n-k) \cosh r=k \cosh r
\end{aligned}
$$

since $H$ is perpendicular to $\partial / \partial r$ on the cone; similarly for a cone $\Sigma \subset S^{n}$.

Given a $(k-1)$-dimensional submanifold $M$ of $S^{n}$ or $H^{n}$ and a point $p$ in $S^{n}$ or $H^{n}$, the $k$-dimensional conc $p \times M$ with center at $p$ is defined to be the set of all minimizing geodesics from $p$ to the points of $M$.

Proposition 1 For any minimal submanifold $\Sigma$ with boundary in $S^{n}$ or $H^{n}$, and any point $p \in S^{n}$ or $H^{n}$,

$$
M_{p}(\Sigma) \leq M_{p}(p \rtimes x \Sigma)
$$

Proof. Let $\nu$ and $\eta$ be the unit conormals to $\partial \Sigma$ on $\Sigma$ and $p \times \partial \Sigma \subset S^{n}$ respectively. Then it is easy to see, as in [4, Proposition 1], that

$$
\frac{\partial r}{\partial \nu} \leq \frac{\partial r}{\partial \eta}
$$

Hence

$$
\begin{aligned}
M_{p}(\Sigma) & =-\frac{1}{k} \int_{\Sigma} \Delta \cos r=\frac{1}{k} \int_{\partial \Sigma} \sin r \frac{\partial r}{\partial \nu} \leq \frac{1}{k} \int_{\partial \Sigma} \sin r \frac{\partial r}{\partial \eta} \\
& =-\frac{1}{k} \int_{p} \Delta \cos r=M_{p}(p \nVdash \partial \Sigma) .
\end{aligned}
$$

The same argument applies to $\Sigma \subset H^{n}$.

Lemma 5 (a) On $S^{n}, \bar{\nabla}^{2} r=\cot r(g-d r \otimes d r)$. On $H^{n}, \bar{\nabla}^{2} r=\operatorname{coth} r(g-d r \otimes d r)$.

(b) Let $\Sigma$ be a k-dimensional minimal submanifold or a cone from $p$ in $S^{n}$ or $H^{n}$. Then $\Delta r=\cot r\left(k-|\nabla r|^{2}\right)$ for $\Sigma \subset S^{n}$, and $\Delta r=\operatorname{coth} r\left(k-|\nabla r|^{2}\right)$ for $\Sigma \subset H^{n}$.

Proof. (a) Using Lemma 3, we compute.

$$
\begin{aligned}
-(\cos r) g & =\bar{\nabla}^{2} \cos r=-\cos r \bar{\nabla} r \otimes \bar{\nabla} r-\sin r \bar{\nabla}^{2} r \\
(\cosh r) g & =\bar{\nabla}^{2} \cosh r=\cosh r \bar{\nabla} r \otimes \bar{\nabla} r+\sinh r \bar{\nabla}^{2} r
\end{aligned}
$$


on $S^{n}$ and $H^{n}$ respectively. The results follow immediately.

(b) From Lemma 4, we have

$$
\begin{aligned}
-k \cos r & =\Delta \cos r=\operatorname{div}(-\sin r \nabla r) \\
& =-\cos r|\nabla r|^{2}-\sin r \Delta r
\end{aligned}
$$

which gives the result; similarly for $\Sigma \subset H^{n}$.

Lemma 6 Let $G(x)$ be Green's function of $S^{k}$ ( $H^{k}$ respectively), whose derivative is $\sin ^{1-k} x$ for $0<x<\pi\left(\sinh ^{1-k} x\right.$ for $0<x<\infty$ respectively). If $\Sigma$ is a $k$-dimensional minimal submanifold of $S^{n}$ (H $H^{n}$ respectively), then $G$ or is subharmonic on $\Sigma-\{ \pm p\}$ $\subset S^{n}$ (on $\Sigma-\{p\} \subset H^{n}$ respectively). (Note: When $k=2, G(r)=G \circ r=\log \tan \frac{r}{2}$, and when $k=3, G(r)=-\cot r$ on $\Sigma \subset S^{n}$.)

Proof.

$$
\begin{aligned}
\Delta G(r) & =\operatorname{div}\left(\sin ^{1-k} r \nabla r\right) \\
& =(1-k) \sin ^{-k} r \cdot \cos r|\nabla r|^{2}+\sin ^{-k} r \cdot \cos r\left(k-|\nabla r|^{2}\right) \\
& =k \sin ^{-k} r \cdot \cos r\left(1-|\nabla r|^{2}\right) \geq 0 .
\end{aligned}
$$

The proof for $\Sigma \subset H^{n}$ is similar.

Definition 2 Let $C \subset S^{\mathbf{n}}$ be a $k$-dimensional rectifiable set and $p$ a point in $S^{\mathbf{n}}$ such that $\operatorname{dist}(p, q)<\pi$ for all $q \in C$. The $k$-dimensional angle $A^{k}(C, p)$ of $C$ viewed from $p$ is defined by setting

$$
A^{k}(C, p)=\sin ^{-k} t \cdot \text { Volume }[(p \nVdash C) \cap S(p, t)]
$$

where $S(p, t)$ is the geodesic sphere of radius $t<\operatorname{dist}(p, C)$ centered at $p$, and the volume is measured counting multiplicity. Clearly the angle does not depend on $t$. There is obviously an analogous definition for the angle of $C \subset H^{n}$ viewed from $p \in H^{n}$.

Note that

$$
A^{k}(C, p)=(k+1) \omega_{k+1} \Theta^{k+1}(p \nVdash C, p),
$$

where $\Theta^{k+1}(p \star C, p)$ is the $(k+1)$-dimensional density of $p \star C$ at $p$. 


\section{Choe - Gulliver}

Proposition 2 Let $\Sigma$ be a $k$-dimensional compact minimal submanifold with boundary in $S^{n}$ or $H^{n}$, and let $p$ be an interior point of $\Sigma$. In case of $\Sigma \subset S^{n}$, we assume that $\operatorname{dist}(p, q) \leq \frac{\pi}{2}$ for all $q \in \Sigma$. Then

$$
A^{k-1}(\partial \Sigma, p) \geq k \omega_{k}
$$

Equality holds if and only if $\Sigma$ is a domain on a totally geodesic submanifold that is star-shaped with respect to $p$.

Proof. Let $B^{n}(p, t) \subset S^{n}$ be the geodesic ball of radius $t$ centered at $p, t<\operatorname{dist}(p, \partial \Sigma)$, $\Sigma_{t}=\Sigma \sim B^{n}(p, t)$, and $C_{t}=\Sigma \cap S(p, t)$. Lemma 6 says that $G(r), r=\operatorname{dist}(p, \cdot)$, is subharmonic. Hence

$$
\begin{aligned}
0 & \leq \int_{\Sigma_{t}} \Delta G(r)=\int_{\Sigma_{t}} \operatorname{div}\left(\sin ^{1-k} r \nabla r\right) \\
& =-\int_{\partial \Sigma_{t}} \sin ^{1-k} r \cdot \frac{\partial r}{\partial \nu}+\int_{\partial \Sigma} \sin ^{1-k} r \cdot \frac{\partial r}{\partial \nu},
\end{aligned}
$$

where $\nu$ on $\partial \Sigma_{t}$ is the unit normal to $\partial \Sigma_{t}$ away from $p$ on $\Sigma \cap B^{n}(p, t)$. Since $\frac{\partial r}{\partial \nu} \rightarrow 1$ and $\frac{\sin t}{t} \rightarrow 1$ on $\partial \Sigma_{t}$ as $t \rightarrow 0$, we have

$$
\begin{aligned}
k \omega_{k} & =\lim _{t \rightarrow 0} \int_{\partial \Sigma} \sin ^{1-k} r \cdot \frac{\partial r}{\partial \nu} \leq \int_{\partial \Sigma} \sin ^{1-k} r \cdot \frac{\partial r}{\partial \nu} \\
& \leq \int_{\partial \Sigma} \sin ^{1-k} r \cdot \frac{\partial r}{\partial \eta}=A^{k-1}(\partial \Sigma, p)
\end{aligned}
$$

where $\eta$ is the same as in Proposition 1. Equality holds if and only if $\Delta G(r)=0$, $\Theta^{k}(\Sigma, p)=1$, and $\nu=\eta$ if and only if $\Sigma$ is a star-shaped minimal cone with density at the center equal to 1 . Since $S^{k-1}$ is the only $(k-1)$-dimensional minimal submanifold in $S^{n}$ with volume $k \omega_{k}$, we conclude that $\Sigma$ lies in a totally geodesic submanifold of $S^{n}$. A similar proof holds for $\Sigma \subset H^{n}$.

\section{Isoperimetric inequality for modified volume}

For a minimal surface $\Sigma$ in $\mathrm{R}^{n}$, the isoperimetric inequality $4 \pi A \leq L^{2}$ has been proved whenever $\partial \Sigma$ is connected, or more generally, whenever there is a point $p \in \mathbf{R}^{n}$ such that no sphere centered at $p$ separates $\partial \Sigma[4]$. We shall prove an analogous result for modified area in $S_{+}^{n}$ :

Theorem 1 Let $\Sigma$ be a compact minimal surface with boundary in $S^{n}$. Assume that $r$ is the distance in $S^{n}$ from a point $p$ of $\Sigma$ and that $r \leq \frac{\pi}{2}$ on $\Sigma$. If $\partial \Sigma$ is radially 
connected from $p$, that is, $\{s: s=\operatorname{dist}(p, q), q \in \partial \Sigma\}$ is a connected interval, then $4 \pi M_{p}(\Sigma) \leq$ Length $(\partial \Sigma)^{2}$. Equality holds if and only if $\Sigma$ is a tolally geodesic disk with center at $p$.

Proof. By Proposition 1, $M_{p}(\Sigma) \leq M_{p}(p \rtimes \partial \Sigma)$. For each geodesic sphere $S(p, t)$ centered at $p$, one has a local isometry between the curve $S(p, t) \cap(p \nVdash \partial \Sigma)$ and a great circle on $S(p, t)$. Therefore, developing $p \star \partial \Sigma$ on a great sphere $S^{2} \subset S^{n}$, one can find a curve $C$ (not necessarily closed) in $S^{2}$ and a local isometry from $p \rtimes \partial \Sigma$ into $\bar{p} \nVdash C$ where $\bar{p}$ is the north pole of $S^{2}$. Clearly we have $M_{p}(p \rtimes \partial \Sigma)=M_{\bar{p}}(\bar{p} \rtimes C)$, Length $(\partial \Sigma)=$ Length $(C)$, and $A^{1}(\partial \Sigma, p)=A^{1}(C, \bar{p})$. Moreover, if we let $\bar{q}$ and $\overline{\bar{q}}$ be the end points of $C$, then $\operatorname{dist}(\bar{p}, \bar{q})=\operatorname{dist}(\bar{p}, \bar{q})$. First, let us assume that $p$ is an interior point of $\Sigma$. We see from Proposition 2 that $A^{1}(\partial \Sigma, p) \geq 2 \pi$ and that $C$ has a self-intersection point. Now, from Lemma 1 , it follows that $M_{\bar{p}}(\bar{p} \rightsquigarrow C)$ equals the Euclidean area of the standard projection of $\bar{p} \nsim C$ onto the plane containing the equator of $S^{2}$. Let $C^{\prime}$ be the image of $C$ under the projection and 0 the origin of the plane. Then $M_{\bar{p}}(\bar{p} \ggg C)=\operatorname{Area}\left(0 ※ C^{\prime}\right), A^{1}(C, \bar{p})=A^{1}\left(C^{\prime}, 0\right)$ and Length $(C) \geq$ Length $\left(C^{\prime}\right)$. The last inequality follows from the fact that the projection is a lengthdecreasing map. Furthermore, if $q^{\prime}$ and $q^{\prime \prime}$ are the end points of $C^{\prime}$, then $\operatorname{dist}\left(0, q^{\prime}\right)=$ $\operatorname{dist}\left(0, q^{\prime \prime}\right)$. Thus we can apply Lemma 1 of $[4]$ and conclude that

$$
4 \pi \operatorname{Area}\left(0 * C^{\prime}\right) \leq \operatorname{Length}\left(C^{\prime}\right)^{2},
$$

and equality holds if and only if $C^{\prime}$ is the boundary of a circle. Therefore

$$
4 \pi M_{p}(\Sigma) \leq \text { Length }(\partial \Sigma)^{2}
$$

and equality holds if and only if Length $\left(C^{\prime}\right)=\operatorname{Length}(\partial \Sigma)$ and $A^{1}(\partial \Sigma, p)=2 \pi$, or equivalently, $\Sigma$ is a totally geodesic disk centered at $p$. Second, if $p$ is a boundary point of $\Sigma$, then we apply Corollary 1 instead of Lemma 1 of [4].

Remark 1 The same inequality is false for minimal surfaces in $H^{n}$. In fact, among domains in $H^{2}$ with prescribed boundary length, the modified area has no upper bound. Our proof fails in $H^{n}$ because the projection in Minkowski space $\mathrm{R}^{n+1}$ from $H^{n}$ onto the hyperplane $x_{n+1}=0$ is a length-increasing map. In Theorem 4 below, however, we shall prove the inequality in the special case that $\partial \Sigma$ lies in a sphere of $H^{n}$ centered at $p$.

$\mathrm{Li}$, Schoen and Yau [7] have proved the isoperimetric inequality for a minimal surface $\Sigma$ in $\mathbf{R}^{n}$, provided that $\partial \Sigma$ is weakly connected, that is, no coordinate hy- 


\section{Choe - Gulliver}

perplane separates $\partial \Sigma$. The analogous hypothesis for $\Sigma^{2}$ in $S^{n}$ suffices to prove an isoperimetric inequality for modified area:

Theorem 2 Let $\Sigma$ be a compact minimal surface wilh boundary in $S^{n} \subset \mathbf{R}^{n+1}$ and $r$ the distance in $S^{n}$ from the north pole $p=(0, \ldots, 0,1) \in S^{n}$. Assume without loss of generality that the center of mass of $\partial \Sigma$ in $\mathbf{R}^{n+1}$ lics on the line $l=\left\{\left(x_{1}, \ldots, x_{n+1}\right)\right.$ : $\left.x_{1}=\cdots=x_{n}=0\right\}$. Assume also that $\partial \Sigma$ is weakly connccled : there exists a rectangular coordinate system $\left\{y_{1}, \ldots, y_{n}\right\}$ in the hyperplane $x_{n+1}=0$ such that, $\mathbf{R}^{n+1}$ being equipped with the coordinate system $\left\{y_{1}, \ldots, y_{n}, x_{n+1}\right\}$, no hyperplanes of $\mathbf{R}^{n+1} y_{i}=$ const, $1 \leq i \leq n$, ever separale $\partial \Sigma$. Then $4 \pi M_{p}(\Sigma) \leq$ Length $(\partial \Sigma)^{2}$.

Proof. Let us modify Li,Schoen and Yau's argument in [7]. Assume $x_{i}=y_{i}(1 \leq i \leq$ $n)$. For any fixed $1 \leq i \leq n$, it follows from the weak connectedness of $\partial \Sigma$ that each component of $\partial \Sigma$ can be translated in a proper direction perpendicular to $\partial / \partial x_{i}$, so that after translation, their union forms a connected one-dimensional simplicial complex $C_{i}$. Observe here that

$$
\int_{\partial \Sigma} x_{i}=\int_{C_{i}} x_{i}, \quad \int_{\partial \Sigma} x_{i}^{2}=\int_{C_{1}} x_{i}^{2}, \quad \int_{\partial \Sigma}\left(\frac{d x_{i}}{d s}\right)^{2}=\int_{C_{i}}\left(\frac{d x_{i}}{d s}\right)^{2},
$$

and $\operatorname{Length}(\partial \Sigma)=\operatorname{Length}\left(C_{i}\right)$.

By hypothesis,

$$
\int_{\partial \Sigma} x^{i}=0, \quad i=1, \ldots, n .
$$

Hence the Poincare inequality applied to $C_{i}$ says that

$$
\int_{C_{i}} x_{i}^{2} \leq \frac{1}{4 \pi^{2}} \operatorname{Length}\left(C_{i}\right)^{2} \int_{C_{\mathrm{i}}}\left(\frac{d x_{i}}{d s}\right)^{2},
$$

where $s$ is the arclength parameter of $C_{i}$. Thus

$$
\begin{aligned}
M_{p}(\Sigma) & =-\int_{\Sigma} \frac{1}{2} \Delta \cos r=\frac{1}{2} \int_{\partial \Sigma} \sin r \frac{\partial r}{\partial \nu} \\
& \leq \frac{1}{2} \int_{\partial \Sigma}|\sin r| \leq \frac{1}{2}\left[\int_{\partial \Sigma} 1\right]^{1 / 2}\left[\int_{\partial \Sigma} \sin ^{2} r\right]^{1 / 2} \\
& =\frac{1}{2} \operatorname{Length}(\partial \Sigma)^{1 / 2}\left[\int_{\partial \Sigma} \sum_{i=1}^{n} x_{i}^{2}\right]^{1 / 2} \\
& \leq \frac{1}{2} \operatorname{Length}(\partial \Sigma)^{1 / 2}\left[\frac{1}{4 \pi^{2}} \sum_{i=1}^{n} \operatorname{Length}\left(C_{i}\right)^{2} \int_{C_{i}}\left(\frac{d x_{i}}{d s}\right)^{2}\right]^{1 / 2} \\
& \leq \frac{1}{4 \pi} \operatorname{Length}(\partial \Sigma)^{3 / 2}\left[\int_{\partial \Sigma} \sum_{i=1}^{n}\left(\frac{d x_{i}}{d s}\right)^{2}\right]^{1 / 2}
\end{aligned}
$$




\section{Choe - Gulliver}

$$
\begin{aligned}
& \leq \frac{1}{4 \pi} \text { Length }(\partial \Sigma)^{3 / 2} \text { Length }(\operatorname{Proj}(\partial \Sigma))^{1 / 2} \\
& \leq \frac{1}{4 \pi} \operatorname{Length}(\partial \Sigma)^{2} .
\end{aligned}
$$

Closely related to isoperimetric inequalities on a minimal submanifold $\Sigma$ is the monotonicity property: the volume of $\Sigma \cap B(p, r)$, divided by the corresponding volume for a tolally gcodesic manifold of the same dimcnsion, is a nondecreasing function of $r$. This property has been proved for $\mathbf{R}^{n}$ and for $H^{n}$ [1], but not for $S^{n}$ (see however [6], p.353). Our next result shows that modified volume enjoys the monotonicity property in all three cases.

Theorem 3 Let $\Sigma$ be a $k$-dimensional minimal submanifold in $S^{\mathbf{n}}$ ( $H^{\mathbf{n}}$ respectively) and $r$ the distance in $S^{n}\left(H^{n}\right.$ respectively) from $p$. Then $\sin ^{-k} r \cdot M_{p}\left(\sum \cap B^{n}(p, r)\right)$ $\left(\sinh ^{-k} r \cdot M_{p}\left(\Sigma \cap B^{n}(p, r)\right)\right.$ respectively) is a monotonically nondecreasing function of $r$ for $0<r<\min \left(\frac{\pi}{2}, \operatorname{dist}(p, \partial \Sigma)\right)(0<r<\operatorname{dist}(p, \partial \Sigma)$ respectively $)$.

Proof. Define $\Sigma_{r}=\Sigma \cap B^{n}(p, r) \subset S^{n}$. Then

$$
\begin{aligned}
M_{p}\left(\Sigma_{r}\right) & =-\frac{1}{k} \int_{\Sigma_{r}} \Delta \cos r \\
& =\frac{1}{k} \int_{\partial \Sigma_{r}} \sin r \frac{\partial r}{\partial \nu}=\frac{1}{k} \sin r \int_{\partial \Sigma_{r}}|\nabla r| .
\end{aligned}
$$

Denote the volume forms on $\Sigma$ and $\partial \Sigma_{r}$ by $d v$ and $d S_{r}$. Then we have $d v=\frac{1}{|\nabla r|} d S_{r} d r$. So

$$
\frac{d}{d r} \int_{\Sigma_{r}} \cos r|\nabla r|^{2} d v=\frac{d}{d r} \int_{0}^{r} \int_{\partial \Sigma_{r}} \cos r|\nabla r| d S_{r} d r=\cos r \int_{\partial \Sigma_{r}}|\nabla r| .
$$

Therefore

$$
\begin{aligned}
M_{p}\left(\Sigma_{r}\right) & =\frac{1}{k} \frac{\sin r}{\cos r} \cos r \int_{\partial \Sigma_{r}}|\nabla r|=\frac{1}{k} \frac{\sin r}{\cos r} \frac{d}{d r} \int_{\Sigma_{r}} \cos r|\nabla r|^{2} \\
& \leq \frac{1}{k} \frac{\sin r}{\cos r} \frac{d}{d r} \int_{\Sigma_{r}} \cos r=\frac{1}{k} \frac{\sin r}{\cos r} \frac{d}{d r} M_{p}\left(\Sigma_{r}\right)
\end{aligned}
$$

In the above inequality we used the fact that $r \leq \frac{\pi}{2}$ and $|\nabla r|^{2} \leq 1$ on $\Sigma$. Hence

$$
\frac{d}{d r} \log \left[\sin ^{-k} r \cdot M_{p}\left(\Sigma_{r}\right)\right] \geq 0
$$

Thus $\sin ^{-k} r \cdot M_{p}\left(\Sigma_{r}\right)$ is nondecreasing; similarly for $\Sigma \subset H^{n}$.

A very special case of the hypothesis of radially connected boundary occurs when a $\Sigma$ lies on a geodesic sphere. In this case, the conclusion of Theorem 1 may be extended to hyperbolic space, and the minimal submanifold may have any dimension. 


\section{Choe - Gulliver}

Theorem 4 Let $\Sigma$ be a $k$-dimensional minimal submanifold in $S^{n}$ or $H^{n}$. Assume that $\partial \Sigma$ lies on a geodesic sphere centercd at a point $p$ of $\Sigma$ and that $r$ is the distance in $S^{n}$ or $H^{n}$ from $p$. Furthermore, in case of $\Sigma \subset S^{n}$, assume $r \leq \frac{\pi}{2}$ on $\Sigma$. Then

$$
k^{k} \omega_{k} M_{p}(\Sigma)^{k-1} \leq \text { Volume }(\partial \Sigma)^{k}
$$

Equality holds if and only if $\Sigma$ is a totally geodesic ball centered at $p$.

Proof. Assume $\Sigma \subset H^{n}$ and let $R$ be the radius of the geodesic sphere in which $\partial \Sigma$ lies. Then

$$
\begin{aligned}
M_{p}(\Sigma) & =\frac{1}{k} \int_{\Sigma} \Delta \cosh r=\frac{1}{k} \int_{\partial \Sigma} \sinh r \frac{\partial r}{\partial \nu} \\
& =\frac{1}{k} \sinh R \int_{\partial \Sigma} \frac{\partial r}{\partial \nu} \leq \frac{1}{k} \sinh R \cdot \operatorname{Volume}(\partial \Sigma)
\end{aligned}
$$

Since $\lim _{r \rightarrow 0} \sinh ^{-k} r \cdot M_{p}\left(\Sigma \cap B^{n}(p, r)\right)=\omega_{k}$, we oblain from Theorem 3,

$$
\sinh ^{-k} R \cdot M_{p}(\Sigma) \geq \omega_{k}
$$

Hence

$$
M_{p}(\Sigma) \leq \frac{1}{k} \omega_{k}^{-1 / k} M_{p}(\Sigma)^{1 / k} \operatorname{Volume}(\partial \Sigma)
$$

and so the desired inequality follows. Obviously equality holds if and only if $\Sigma$ is a cone with density at the center equal to 1 , or equivalently, $\Sigma$ is a totally geodesic ball. A similar proof holds for $\Sigma \subset S^{n}$.

\section{Isoperimetric inequalities via Fubini's theorem}

All the theorems of preceding section require that $\partial \Sigma$ satisfy some hypotheses related to connectivity. Without such hypotheses in $\mathbf{R}^{n}$, the strongest inequality known for minimal surfaces is due to Leon Simon. Simon used Fubini's theorem to obtain a weaker isoperimetric inequality for a minimal surface in $\mathbf{R}^{n}$ (see $[3, \mathrm{p} .318]$, [8, Theorem 5.3]) :

$$
2 \pi \operatorname{Area}(\Sigma) \leq \text { Length }(\partial \Sigma)^{2} .
$$

The key idea, rephrased in our setting, is to integrate the angle of $\partial \Sigma$ from the points all over $\Sigma$. In this section we extend his method from $\mathbf{R}^{n}$ to $S^{n}$ and $H^{n}$, and derive various isoperimetric inequalities in space forms. Let us first reprove Simon's theorem. 


\section{Choe - Gulliver}

Lemma 7 (a) Let $D$ and $g$ be the Euclidean connection and the metric respectively, and let $r$ be the distance in $\mathbf{R}^{n}$ from a fixed point $p$. Then

$$
D^{2} r=\frac{1}{r}(g-d r \otimes d r) \text {. }
$$

(b) $D^{2} r^{2}=2 g$.

(c) Let $\Sigma$ be a $k$-dimensional minimal submanifold of $\mathbf{R}^{n}$. Then

$$
\Delta r^{\alpha}=\alpha r^{\alpha-2}\left(k+(\alpha-2)|\nabla r|^{2}\right)
$$

(d) $\Delta \log r=\frac{1}{r^{2}}\left(k-2|\nabla r|^{2}\right)$.

Proof. Conclusions (a) and (b) are standard computations in orthogonal coordinates of $\mathrm{R}^{n}$. Conclusions (c) and (d) follow from Lemma 2 and straightforward computations.

Proof of Simon's theorem. By the above lemma, $\log r$ is subharmonic on a minimal surface $\Sigma$. From [4, Proposition 2], or by following the same argument as in Proposition 2 of this paper, we have

$$
2 \pi \leq A^{1}(\partial \Sigma, p)
$$

where $p$ is an interior point of $\Sigma$.

More precisely,

$$
2 \pi \leq \int_{\partial \Sigma} \frac{1}{r_{p}} \frac{\partial r}{\partial \nu} \leq \int_{\partial \Sigma} \frac{1}{r_{p}}
$$

where $r_{p}$ indicates that the distance $r$ is measured from a fixed $p$. Letting $p$ range over all points of $\Sigma$ and integrating the above inequality over $\Sigma$, we get

$$
2 \pi \operatorname{Area}(\Sigma) \leq \int_{\Sigma} \int_{\partial \Sigma} \frac{1}{r_{p}} .
$$

Then by Fubini's theorem,

$$
2 \pi \operatorname{Area}(\Sigma) \leq \int_{\partial \Sigma} \int_{\Sigma} \frac{1}{r_{p}}
$$

Now by Lemma $\gamma(c)$ with $\alpha=1$,

$$
\Delta r \geq \frac{1}{r}
$$

Hence

$$
2 \pi \operatorname{Area}(\Sigma) \leq \int_{\partial \Sigma} \int_{\Sigma} \Delta r=\int_{\partial \Sigma} \int_{\partial \Sigma} \frac{\partial r}{\partial \nu} \leq \int_{\partial \Sigma} \int_{\partial \Sigma} 1=\operatorname{Length}(\partial \Sigma)^{2},
$$




\section{Choe - Gulliver}

which completes the proof.

The key point in this proof is the nice relationship between the gradient of $\log r$ and the Laplacian of $r$. In order to apply this method to a minimal surface in a space form, we need to choose suitable functions of $r$ and compute their Laplacians.

Lemma 8 Let $\Sigma$ be a $k$-dimensional minimal submanifold in $S^{n}$.

(a) $\Delta \log \sin r=k \cot ^{2} r-\frac{|\nabla r|^{2}}{\sin ^{2} r}\left(1+\cos ^{2} r\right)$.

(b) $\Delta \log \cos r=-k-|\nabla r|^{2} \tan ^{2} r$.

Assume $\Sigma \subset H^{n}$.

(c) $\Delta \log \sinh r=k \operatorname{coth}^{2} r-\frac{|\nabla r|^{2}}{\sinh ^{2} r}\left(1+\cosh ^{2} r\right)$.

(d) $\Delta \log \cosh r=k-|\nabla r|^{2} \tanh ^{2} r$.

Proof. (a)

$$
\begin{aligned}
\Delta \log \sin r & =\operatorname{div}\left(\frac{\cos r}{\sin r} \nabla r\right) \\
& =-\frac{1}{\sin ^{2} r}|\nabla r|^{2}+\frac{\cos r}{\sin r} \Delta r \\
& =-\frac{1}{\sin ^{2} r}|\nabla r|^{2}+\frac{\cos ^{2} r}{\sin ^{2} r}\left(k-|\nabla r|^{2}\right) \\
& =k \frac{\cos ^{2} r}{\sin ^{2} r}-\frac{|\nabla r|^{2}}{\sin ^{2} r}\left(1+\cos ^{2} r\right) .
\end{aligned}
$$

Here we used Lemma $5(b)$ for $\Delta r$.

(b)

$$
\begin{aligned}
\Delta \log \cos r & =\operatorname{div}\left(-\frac{\sin r}{\cos r} \nabla r\right)=-\frac{1}{\cos ^{2} r}|\nabla r|^{2}-\frac{\sin r}{\cos r} \Delta r \\
& =-k+|\nabla r|^{2}\left(1-\frac{1}{\cos ^{2} r}\right) .
\end{aligned}
$$

A similar proof holds for $\Sigma \subset H^{n}$.

Lemma 9 On a $k$-dimensional minimal submanifold $\Sigma$ of $S^{n}$,

$$
\Delta \sin r=\frac{1}{\sin r}\left(k \cos ^{2} r-|\nabla r|^{2}\right) .
$$

Proof.

$$
\begin{aligned}
\Delta \sin r & =\operatorname{div}(\cos r \nabla r)=-\sin r|\nabla r|^{2}+\cos r \Delta r \\
& =-\frac{\sin ^{2} r+\cos ^{2} r}{\sin r}|\nabla r|^{2}+k \frac{\cos ^{2} r}{\sin r} \\
& =\frac{1}{\sin r}\left(k \cos ^{2} r-|\nabla r|^{2}\right) .
\end{aligned}
$$




\section{Choe - Gulliver}

Theorem $5(a)$ Let $\Sigma$ be a minimal surface in $S^{n}$ with $\operatorname{diam}(\Sigma) \leq \frac{\pi}{2}$. Let $A=$ Area $(\Sigma)$ and $L=$ Length $(\partial \Sigma)$. Then

$$
2 \pi A \leq L^{2}+A^{2}
$$

(b) For a minimal surface $\Sigma$ in $H^{n}$,

$$
2 \pi A \leq L^{2}-A^{2}
$$

Proof. From Lemma $8(a)$ we have

$$
\begin{aligned}
\Delta \log \sin r & =\frac{2 \cos ^{2} r}{\sin ^{2} r}-\frac{|\nabla r|^{2}}{\sin ^{2} r}\left(1+\cos ^{2} r\right) \\
& =\frac{\cos ^{2} r}{\sin ^{2} r}+\frac{1-\sin ^{2} r}{\sin ^{2} r}-\frac{|\nabla r|^{2}}{\sin ^{2} r}\left(1+\cos ^{2} r\right) \\
& =\frac{1-|\nabla r|^{2}}{\sin ^{2} r}\left(1+\cos ^{2} r\right)-1 \geq-1 .
\end{aligned}
$$

Therefore, if we choose $r=r_{p}$ where $p \in \Sigma$, we find

$$
\begin{aligned}
-A & =\int_{\Sigma}-1 \leq \int_{\Sigma} \Delta \log \sin r \\
& =-\lim _{t \rightarrow 0} \int_{\partial \Sigma_{t}} \frac{\cos r}{\sin r} \frac{\partial r}{\partial \nu}+\int_{\partial \Sigma} \frac{\cos r}{\sin r} \frac{\partial r}{\partial \nu},
\end{aligned}
$$

where $\nu$ on $\partial \Sigma_{t}$ is the outer conormal on $\Sigma \cap B^{n}(p, t)$. Note that on $\partial \Sigma_{t}$,

$$
r \frac{\cos r}{\sin r} \rightarrow 1 \text { and } \quad \frac{\partial r}{\partial \nu} \rightarrow 1 \quad \text { as } t \rightarrow 0 \text {. }
$$

Hence for $r \leq \frac{\pi}{2}$,

$$
-A \leq-2 \pi+\int_{\partial \Sigma} \frac{\cos r_{p}}{\sin r_{p}}
$$

where $r_{p}$ is the distance from a fixed point $p$. Let $p$ range over $\Sigma$ and integrate both sides of the inequality over $\Sigma$ :

$$
-A^{2} \leq \int_{\Sigma}-2 \pi+\int_{\Sigma} \int_{\partial \Sigma} \frac{\cos r_{p}}{\sin r_{p}}=-2 \pi A+\int_{\partial \Sigma} \int_{\Sigma} \frac{\cos r_{p}}{\sin r_{p}}
$$

By Lemma $5(b)$,

$$
\Delta r \geq \cot r
$$

Hence

$$
2 \pi A-A^{2} \leq \int_{\partial \Sigma} \int_{\Sigma} \Delta r=\int_{\partial \Sigma} \int_{\partial \Sigma} \frac{\partial r}{\partial \nu} \leq L^{2} .
$$

(b) Lemma 8 (c) states that

$$
\begin{aligned}
\Delta \log \sinh r & =\frac{2 \cosh ^{2} r}{\sinh ^{2} r}-\frac{|\nabla r|^{2}}{\sinh ^{2} r}\left(1+\cosh ^{2} r\right) \\
& \geq \frac{2 \cosh ^{2} r-1-\cosh ^{2} r}{\sinh ^{2} r}=1 .
\end{aligned}
$$


Hence

$$
A \leq \int_{\Sigma} \Delta \log \sinh r \leq-2 \pi+\int_{\partial \Sigma} \frac{\cosh r}{\sinh r}
$$

and

$$
\begin{aligned}
A(A+2 \pi) & \leq \int_{\Sigma} \int_{\partial \Sigma} \frac{\cosh r}{\sinh r}=\int_{\partial \Sigma} \int_{\Sigma} \frac{\cosh r}{\sinh r} \\
& \leq \int_{\partial \Sigma} \int_{\Sigma} \Delta r=\int_{\partial \Sigma} \int_{\partial \Sigma} \frac{\partial r}{\partial \nu} \leq L^{2} .
\end{aligned}
$$

Remark 2 Evidently, one can rewrite Simon's theorem and Theorem $5(a),(b)$ in a single form

$$
2 \pi A \leq L^{2}+K A^{2}
$$

where $K$ is the sectional curvature of the space form that the minimal surface $\Sigma$ sits in.

Due to the analytic nature of the proof of Theorem 5, we can derive, applying the same argument, the Sobolev-type inequalities corresponding to the above isoperimetric inequalities. As is well known, one can recover the isoperimetric inequalities from the Sobolev-type inequalities, using smoothed characteristic functions as test functions.

Corollary 1 Let $f$ be a compactly supported smooth nonncgative function on a minimal surface $\Sigma$ in $S^{n}$ or $H^{n}$. In case $\Sigma \subset S^{n}$, assume also that the diameter in $S^{n}$ of the support of $f$ is $\leq \frac{\pi}{2}$. Then

$$
2 \pi \int_{\Sigma} f^{2} \leq\left(\int_{\Sigma}|\nabla f|\right)^{2}+K\left(\int_{\Sigma} f\right)^{2}
$$

where $K=1$ or -1 depending on whether $\Sigma \subset S^{n}$ or $\Sigma \subset H^{n}$.

Proof. For $\Sigma \subset S^{n}$, we have

$$
\operatorname{div}(f \nabla \log \sin r) \geq\langle\nabla f, \cot r \nabla r\rangle-f
$$

Integrating both sides, we see that for a fixed $y \in \Sigma$,

$$
2 \pi f(y) \leq \int_{\Sigma}|\nabla f(x)| \cdot \cot r_{y}(x) d A_{x}+\int_{\Sigma} f(x) d A_{x}
$$

where $r_{y}(x)=\operatorname{dist}(x, y)$. Moreover

$$
\operatorname{div}(f \nabla r) \geq\langle\nabla f, \nabla r\rangle+f \cot r
$$




\section{Choe - Gulliver}

So

$$
\int_{\Sigma} f(y) \cot r_{x}(y) d A_{y} \leq \int_{\Sigma}|\nabla f(y)| d A_{y}
$$

Therefore

$$
\begin{aligned}
2 \pi \int_{\Sigma} f^{2} & \leq \int_{\Sigma} f(y)\left(\int_{\Sigma}|\nabla f(x)| \cdot \cot r_{y}(x) d A_{x}+\int_{\Sigma} f(x) d A_{x}\right) d A_{y} \\
& =\int_{\Sigma}|\nabla f(x)|\left(\int_{\Sigma} f(y) \cot r_{x}(y) d A_{y}\right) d A_{x}+\left(\int_{\Sigma} f\right)^{2} \\
& \leq\left(\int_{\Sigma}|\nabla f|\right)^{2}+\left(\int_{\Sigma} f\right)^{2} .
\end{aligned}
$$

A similar proof is valid for $\Sigma \subset H^{n}$.

The inequality of part (b) of the following theorem was proved for domains in $H^{k}$ by Yau [10, p.498]. Although it is not sharp, it may be observed that the inequality is asymptotically sharp, by considering geodesic balls in $H^{k}$ of large radius.

Theorem 6 (a) Let $\Sigma$ be a domain in $S^{k}$ or a $k$-dimensional minimal submanifold of $S^{n}$. Assume $\operatorname{diam}(\Sigma) \leq d \leq \frac{\pi}{2}$. Then

$$
k \cdot \operatorname{Volume}(\Sigma) \leq \tan d \cdot \operatorname{Volume}(\partial \Sigma)
$$

(b) Let $\Sigma$ be a domain in $H^{k}$ or a $k$-dimensional minimal submanifold of $H^{n}$. Then

$$
(k-1) \text { Volume }(\Sigma) \leq \text { Volume }(\partial \Sigma)
$$

Proof. (a) Lemma $8(b)$ says that

$$
-\Delta \log \cos r=k-|\nabla r|^{2}\left(1-\frac{1}{\cos ^{2} r}\right) \geq k \text {. }
$$

Therefore

$$
k \cdot \operatorname{Volume}(\Sigma) \leq \int_{\Sigma}-\Delta \log \cos r \leq \int_{\partial \Sigma} \tan d=\tan d \cdot \text { Volume }(\partial \Sigma) .
$$

(b) From Lemma $8(d)$,

$$
\Delta \log \cosh r=k+|\nabla r|^{2}\left(\frac{1}{\cosh ^{2} r}-1\right) \geq k-|\nabla r|^{2} \geq k-1 .
$$

So

$$
(k-1) \text { Volume }(\Sigma) \leq \int_{\Sigma} \Delta \log \cosh r \leq \int_{\partial \Sigma} \frac{\sinh r}{\cosh r} \leq \int_{\partial \Sigma} 1=\text { Volume }(\partial \Sigma)
$$




\section{Choe - Gulliver}

Corollary 2 (a) Let $f$ be a nonnegative smooth function with compact support on a $k$-dimensional minimal submanifold $\Sigma$ in $S^{n}$. If $\operatorname{diam}(s p t f) \leq d \leq \frac{\pi}{2}$, then

$$
k \int_{\Sigma} f \leq \tan d \int_{\Sigma}|\nabla f|
$$

(b) If $\Sigma^{k} \subset H^{n}$, then

$$
(k-1) \int_{\Sigma} f \leq \int_{\Sigma}|\nabla f|
$$

Proof. (b) From Lemma 5(b), we have

$$
\operatorname{div}(f \nabla r) \geq\langle\nabla f, \nabla r\rangle+(k-1) f .
$$

Integrating both sides over $\Sigma$ gives the desired inequality. For $(a)$, we obscrve

$$
\operatorname{div}(f \nabla \log \cos r) \leq-\langle\nabla f, \tan r \nabla r\rangle-k f
$$

Theorem 7 Let $\Sigma$ be a minimal surface in $S^{n}$.

(a) If $\operatorname{diam}(\Sigma) \leq d \leq \frac{\pi}{2}$ and $c=\cos d$, then

$$
2 \pi c A \leq L^{2}
$$

(b) If $\operatorname{diam}(\Sigma) \leq \frac{\pi}{2}$, then

$$
2 \pi A \leq 2 A L+L^{2}
$$

Proof. In the proof of Proposition 2, we obtained

$$
2 \pi \leq \int_{\partial \Sigma} \frac{1}{\sin r} \frac{\partial r}{\partial \nu} \leq \int_{\partial \Sigma} \frac{1}{\sin r}
$$

if $\operatorname{dist}(p, \partial \Sigma) \leq \frac{\pi}{2}$. From Lemma $5(b)$, we have for $r \leq d$

$$
\Delta r \geq \frac{c}{\sin r}
$$

Hence, if $\operatorname{diam}(\Sigma) \leq \frac{\pi}{2}$,

$$
\begin{aligned}
2 \pi A & \leq \int_{\Sigma} \int_{\partial \Sigma} \frac{1}{\sin r}=\int_{\partial \Sigma} \int_{\Sigma} \frac{1}{\sin r} \\
& \leq \int_{\partial \Sigma} \int_{\Sigma} \frac{1}{c} \Delta r \leq \int_{\partial \Sigma} \int_{\partial \Sigma} \frac{1}{c}=\frac{L^{2}}{c} .
\end{aligned}
$$

This proves $(a)$. For $(b)$, we use Lemma 9 and obtain

$$
\Delta \sin r \geq \frac{2 \cos ^{2} r-1}{\sin r}=\frac{1}{\sin r}-2 \sin r \geq \frac{1}{\sin r}-2 .
$$




\section{Choe - Gulliver}

Therefore

$$
\begin{aligned}
2 \pi A & \leq \int_{\partial \Sigma} \int_{\Sigma} \frac{1}{\sin r} \leq \int_{\partial \Sigma} \int_{\Sigma}(2+\Delta \sin r) \\
& \leq \int_{\partial \Sigma}\left(2 A+\int_{\partial \Sigma} \cos r\right) \leq 2 A L+\int_{\partial \Sigma} \int_{\partial \Sigma} 1 \\
& =2 A L+L^{2}
\end{aligned}
$$

Corollary 3 Assume that $\Sigma$ is a minimal surface in $S^{n}$ and that $f \in C_{0}^{\infty}(\Sigma)$ and $f \geq 0$.

(a) If $\operatorname{diam}(\operatorname{sptf}) \leq d \leq \frac{\pi}{2}$ and $c=\cos d$, then

$$
2 \pi c \int_{\Sigma} f^{2} \leq\left(\int_{\Sigma}|\nabla f|\right)^{2}
$$

(b) If $\operatorname{diam}(\operatorname{sptf}) \leq \frac{\pi}{2}$, then

$$
2 \pi \int_{\Sigma} f^{2} \leq\left(\int_{\Sigma}|\nabla f|\right)^{2}+2\left(\int_{\Sigma}|\nabla f|\right) \cdot\left(\int_{\Sigma} f\right)
$$

Proof. (a) From Lemma 5(b) it follows that

$$
\begin{aligned}
\operatorname{div}\left(f \frac{1}{\sin r} \nabla r\right) & =\left\langle\nabla f, \frac{1}{\sin r} \nabla r\right\rangle+2 f \frac{\cos r}{\sin ^{2} r}\left(1-|\nabla r|^{2}\right) \\
& \geq\left\langle\nabla f, \frac{1}{\sin r} \nabla r\right\rangle .
\end{aligned}
$$

Hence

Similarly,

$$
2 \pi f(y) \leq \int_{\Sigma} \frac{|\nabla f(x)|}{\sin r_{y}(x)} d A_{x}
$$

and hence

$$
\operatorname{div}(f \nabla r) \geq\langle\nabla f, \nabla r\rangle+\frac{c f}{\sin r}
$$

Thus

$$
\int_{\Sigma} \frac{c f(y)}{\sin r_{x}(y)} d A_{y} \leq \int_{\Sigma}|\nabla f(y)| d A_{y}
$$

$$
\begin{aligned}
2 \pi \int_{\Sigma} f^{2} & \leq \int_{\Sigma} f(y)\left(\int_{\Sigma} \frac{|\nabla f(x)|}{\sin r_{y}(x)} d A_{x}\right) d A_{y} \\
& =\int_{\Sigma}|\nabla f(x)|\left(\int_{\Sigma} \frac{f(y)}{\sin r_{x}(y)} d A_{y}\right) d A_{x} \leq \frac{1}{c}\left(\int_{\Sigma}|\nabla f|\right)^{2} .
\end{aligned}
$$

(b) Since

$$
\operatorname{div}(f \nabla \sin r) \geq\langle\nabla f, \cos r \nabla r\rangle+f\left(\frac{1}{\sin r}-2\right)
$$




\section{Choe - Gulliver}

we obtain

$$
\int_{\Sigma} \frac{f(y)}{\sin r_{x}(y)} d A_{y} \leq \int_{\Sigma}|\nabla f(y)| d A_{y}+2 \int_{\Sigma} f(y) d A_{y} .
$$

Therefore as in $(a)$,

$$
\begin{aligned}
2 \pi \int_{\Sigma} f^{2} & \leq \int_{\Sigma}|\nabla f(x)|\left(\int_{\Sigma} \frac{f(y)}{\sin r_{x}(y)} d A_{y}\right) d A_{x} \\
& \leq \int_{\Sigma}|\nabla f(x)|\left(\int_{\Sigma}|\nabla f(y)| d A_{y}+2 \int_{\Sigma} f(y) d A_{y}\right) d A_{x} \\
& =\left(\int_{\Sigma}|\nabla f|\right)^{2}+2\left(\int_{\Sigma}|\nabla f|\right) \cdot\left(\int_{\Sigma} f\right) .
\end{aligned}
$$

\section{References}

[1] M. Anderson, Complete minimal varieties in hyperbolic space. Invent. Math. 69 $477-494(1982)$

[2] F. Bernstein, Über die isoperimetrische Eigenschaft des Kreises auf der Kugeloberfläche und in der Ebene. Math. Ann. 60, 117-136 (1905)

[3] E. Bombieri, An introduction to minimal currents and parametric variational problems. Mathematical Reports 2, Chur-London-Paris-New York: Harwood Academic 1985 Academic Publishers (1985)

[4] J. Choe, The isoperimetric inequality for a minimal surface with radially connected boundary. Ann. Scuola Norm. Sup. Pisa Cl. Sci. (4), 17, 583-593 (1990)

[5] J. Choe and R. Gulliver, The sharp isoperimetric inequality for minimal surfaces with radially connected boundary in hyperbolic space. to appear in Invent. Math.

[6] R. Gulliver and P. Scott, Least area surfaces can have excess triple points. Topology 26, 345-359 (1987)

[7] P. Li, R. Schoen, and S.-T. Yau, On the isoperimetric inequality for minimal surfaces. Ann. Scuola Norm. Sup. Pisa Cl. Sci. (4), 11, $23 i \cdot 244$ (1984)

[8] R. Osserman, The isoperimetric inequality. Bull. Amer. Math. Soc. 84, 11821238 (1978) 


\section{Choe - Gulliver}

[9] E. Schmidt, Über die isoperimetrische Aufgabe im n-dimensionalen Raum konstanter negativer Krümmung. I. Die isoperimetrischen Ungleichungen in der hyperbolischen Ebene und für Rotationskörper im n-dimensionalen hyperbolischen Raum. Math. Z. 46, 204-230 (1940)

[10] S.-T. Yau, Isoperimetric constants and the first eigenvalue of a compact manifold. Ann. Sci. Ecole Norm. Sup. 8, 487-507 (1975)

Jaigyoung Choe

Department of Mathematics

Postech, P.O.Box 125

Pohang, South Korea

Robert Gulliver

School of Mathematics

University of Minnesota

Minneapolis, Minnesota 55455, USA

(Recelved March 6, 1992) 